\title{
Continuity of the nucleation of bulk and surface phases
}

\author{
Richard P. Sear ${ }^{\mathrm{a})}$ \\ Department of Physics, University of Surrey Guildford, Surrey GU2 7XH, United Kingdom
}

(Received 4 August 2008; accepted 10 September 2008; published online 28 October 2008)

\begin{abstract}
Nucleation of a new bulk phase is almost always heterogeneous: It occurs at a surface. Nucleation of a new surface phase also occurs at a surface of course. We study nucleation in an Ising model with both bulk and surface (prewetting) transitions. We find no clear distinction between nucleation of the bulk and surface phases. As the bulk transition is crossed, the phase that is nucleating changes from being a new surface phase to being a new bulk phase. However, neither the nucleation rate nor the nucleus itself changes discontinuously. It appears that because the nucleus is only approximately two spins high, it is oblivious to the fact that on one side of the bulk transition it will grow to form a thick wetting layer, while on the other side it will grow to form a bulk phase of limitless thickness. The phase diagram of hard spheres at a hard wall is analogous to that of our model. Therefore, we suggest that the nucleation of the crystal phase of hard spheres at a hard wall may also be indistinguishable from that associated with a prefreezing surface transition. (C) 2008 American Institute of Physics. [DOI: 10.1063/1.2992160]
\end{abstract}

\section{INTRODUCTION}

The nucleation of a new bulk phase almost always occurs via heterogeneous nucleation: the nucleus of the new phase forms not in the bulk but at a surface. ${ }^{1}$ The barrier to nucleation is typically much lower at the surface than in the bulk. Because nucleation occurs at a surface, the surfaces present can control how a new bulk phase nucleates. Now, bulk phase transitions are not the only phase transitions; there are also surface phase transitions. Of course, nucleation at a first-order surface transition will also occur at a surface. Here we consider a simple system with both a bulk and a surface transition, and find that near the bulk transition the nuclei of the new surface and bulk phases appear indistinguishable. Bulk and surface phases are clearly distinct: a surface phase transition only affects the surface-it does not reach into the bulk. However, the nucleus of a new phase is only microscopic; indeed they are typically only a few molecules or spins across. Thus it appears that this small nucleus does not "know" whether it will grow to form a thick wetting layer or a bulk phase (both are much thicker than it is).

Simulation snapshots of nuclei for bulk and surface transitions are shown in Fig. 1. They are very similar. We expect that the continuity of the nucleation of surface and bulk transitions is generic. Therefore we have chosen to simulate perhaps the simplest model system. This is the threedimensional Ising model; ${ }^{2,3}$ we take a variant with a surface field and with a coupling between spins at the surface that is different from that in the bulk. See Refs. 4-6 for earlier work on the phase diagram of this model. In the following section we will describe both the model and our simulation methodology. In Sec. III we describe the equilibrium phase behavior of this model at the one temperature we study. This is a

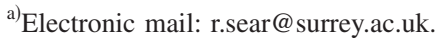

necessary prerequisite to a study of nucleation. Then in Sec. IV we present our detailed results for nucleation. Our final section is a conclusion.

We were inspired to work on nucleation in a system with bulk and surface phase transitions, by work on hard spheres at a smooth hard wall. There the transitions are not between spin-down and spin-up phases but between fluid and crystal
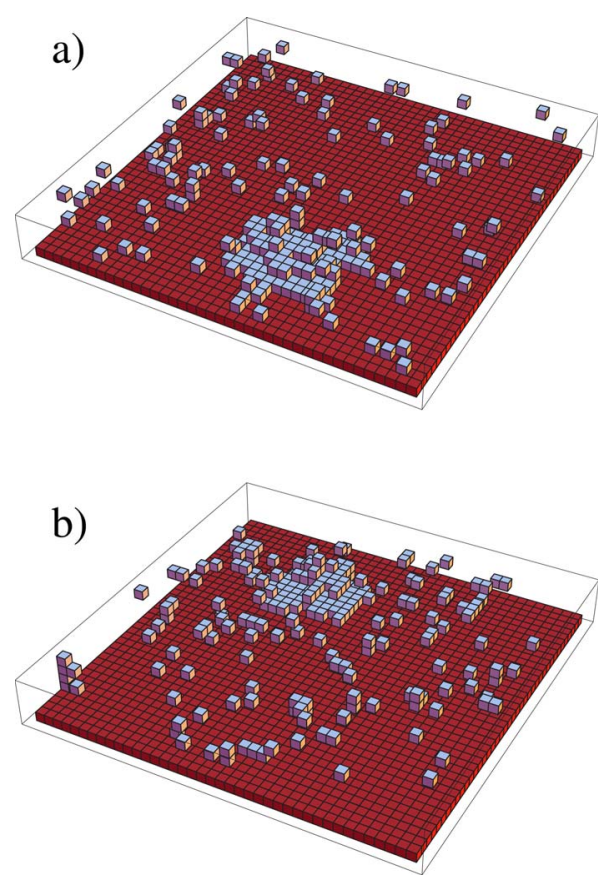

FIG. 1. (Color online) Snapshots of nuclei near the top of the barrier, i.e., of nuclei which have nearly equal probabilities of going forward into the new phase or of falling back into the old phase. (a) and (b) are at bulk fields $h / k T=-0.005$ and 0.005 , respectively, and so in (a) a surface phase is nucleating while in (b) a bulk phase is nucleating. The snapshots are of systems with $L=40$ and $W=10$, but for clarity we only show spins in the first four layers, $z \leq 4$. The wall at $z=0$ is shown as a solid monolayer of dark red cubes while the lattice sites with up spins are shown as pale cubes. Down spins are not shown for clarity. 
phases. However, the phase behavior appears to be analogous to that of our Ising model. Hard spheres at a hard wall have been extensively studied over the past 15 years, starting with the pioneering work of Courtemanche et al. ${ }^{7,8}$ More recent work by Dijkstra and co-workers ${ }^{9-11}$ and by Laird and co-workers ${ }^{12-14}$ focused on whether or not wetting occurs, and also found hysteresis (evidence for a first-order surface transition). In addition Auer and Frenkel ${ }^{15}$ studied nucleation of the bulk crystal at a hard wall. If the analogy between the phase behavior in the two systems extends to the nucleation behavior, then the results we obtain here can be used to predict the qualitative nature of nucleation of bulk and surface crystalline phases of hard spheres at a hard wall.

\section{MODEL AND SIMULATION TECHNIQUE}

We are interested in a generic question, which is as follows. Consider a system with nearby first-order surface and bulk phase transitions. In this system consider the nucleation of both the new bulk phase and of the new surface phase, which looks approximately like a few layers of the new bulk phase. Now, if we vary a parameter such as the magnetic field, or the temperature, we can cross the bulk transition at which point the new phase nucleating changes discontinuously from being a new surface phase to being a new bulk phase. The question then is: Does the nucleus and nucleation rate change discontinuously when the phase that is formed changes? We will show that within the precision of our calculations, the answer is no.

As we are interested in a generic question, we should be able to choose any convenient model. Therefore, we take perhaps the simplest model that has first-order bulk and surface phase transitions. This is the Ising model. We study the three-dimensional Ising model on a simple cubic lattice with nearest-neighbor interactions. ${ }^{2,3,16}$

We study lattices of size $L \times L \times W$. For our calculations of the nucleation rate, we consider a lattice with one wall that favors the spin-up phase and one wall that favors the spindown phase. These walls are flat and the wall that favors the spin-up phase is the $z=0$ plane. For our calculations of isotherms, we consider a symmetric system in which both walls favor the spin-up phase. These walls are the $z=0$ and $z=W$ +1 planes. There are periodic boundary conditions in the $x$ and $y$ directions. For nucleation calculations we take $W$ large enough that nucleation on the wall at $z=0$ is unaffected by the wall at $z=W+1$. For isotherm calculations we take $W$ large enough that the surface phases on the two walls are far enough apart that they are independent.

In the bulk the Ising model is a textbook model. ${ }^{2,3}$ There, neighboring spins interact with a coupling of $J$ and feel a bulk field $h$. At a wall that favors the spin-up phase, the spins feel a surface field $h_{s}$. This is in addition to $h$ and acts only on spins immediately adjacent to the wall. Pairs of spins at walls that favor the spin-up phase also have a different coupling, $J_{s}$ not $J$. For a wall that favors the spin-down phase, we leave the spin coupling at its value in the bulk and impose a surface field of $-J$ to favor the spin-down phase. This eliminates nucleation at these walls. This extension of the bulk Ising model to study surface phase behavior was previously studied by Binder and co-workers. ${ }^{4-6}$

We run our simulations at constant values of fields $h$ and $h_{s}$, and variable numbers of up spins. Then the appropriate weight of a state is $\exp (-H / k T)$, with $H\left(h, h_{s}, J, J_{s}\right)$ given by

$$
H=-J \sum_{i, j}{ }^{\prime} S_{i} S_{j}-J_{s} \sum_{i, j}{ }^{\prime \prime} S_{i} S_{j}-h \sum_{i} S_{i}-h_{s} \sum_{i}{ }^{\prime \prime \prime} S_{i},
$$

where $S_{i}= \pm 1$ is the spin on site $i$. The sum with one prime is over all pairs of neighboring spins, except those in which both spins are next to a wall. The sum with two primes is over all pairs of spins, both of which are adjacent to a wall. Finally, the sum with three primes is over all spins adjacent to a wall.

\section{A. Simulation of nucleation}

Our simulations are with the standard Glauber Metropolis Monte Carlo dynamics. We pick a spin at random and attempt to flip it. If this flip decreases $H$, we always accept it; if the change in $H, \Delta H$, is positive, we accept the spin flip with probability $\exp (-\Delta H / k T)$.

It is not practicable to run simulations for long enough to observe nucleation when the nucleation rate is small. Thus we use the recently developed forward flux sampling (FFS) algorithm of Allen et al. ${ }^{17,18}$ This provides a reasonably simple way to calculate the rate $R_{A B}$ of an activated process where a system starts in one phase, call it phase $A$, then transforms to another phase, phase $B$. Phase $A$ is with the bulk in the spin-down phase and the surface in the metastable state without the prewetting transition having occurred. A surface in this state is shown in Fig. 2(a). For $h$ $<0$, phase $B$ is a state with the bulk in the spin-down phase but with the prewetting surface transition having occurred. This state is shown in Fig. 2(b). For $h>0$, phase $B$ is a state with the bulk in the spin-up phase.

So that the rate is well defined, we need to precisely define phases $A$ and $B$. The spin-down phase will have only a few up spins; the vast majority of the spins will be down spins. When either phase nucleates the number of up spins will increase dramatically. Thus the total number of up spins in the system varies rapidly as either transition takes place and so it makes a good order parameter; we denote it by $\lambda$. Then we define that phase $A$ is being composed of all states with no more than $\lambda_{A}$ up spins, while phase $B$ consists of all states with at least $\lambda_{B} \gg \lambda_{A}$ up spins. Here we define $\lambda_{B}$ to be sufficiently large that there is only a negligible probability of returning to phase $A$. However, it is small enough that the nucleating surface and bulk phases both have $\gg \lambda_{B}$ up spins. Thus the value of $\lambda_{B}$ does not distinguish between the surface and bulk phases. We also run on some simulations to determine which final phase forms, whether it is a new surface or bulk phase.

Having defined our order parameter, our implementation of the FFS algorithm is standard and essentially identical to that in our earlier work. ${ }^{19}$ In particular we use the same order parameter. See Refs. 17-19 for further details of the algorithm. 

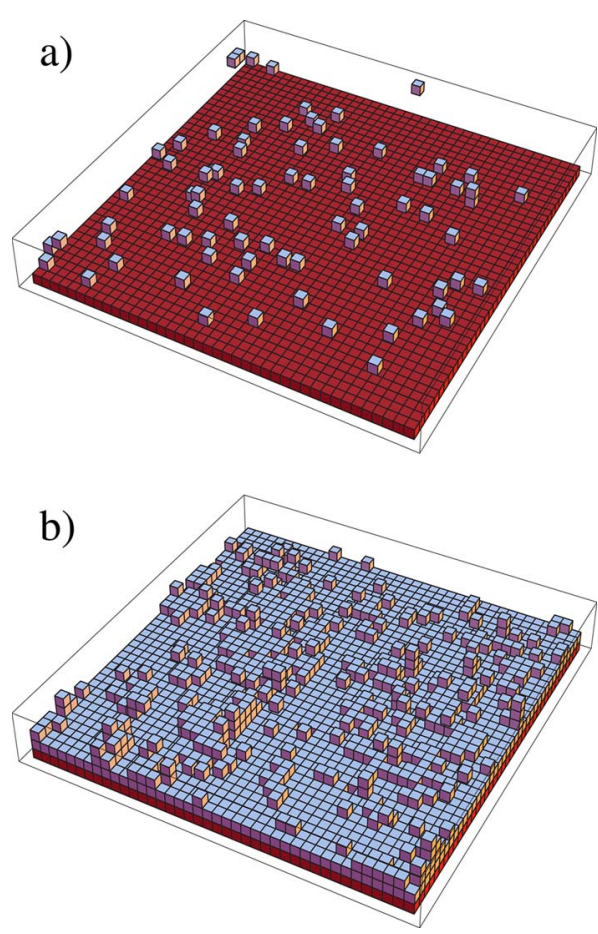

FIG. 2. (Color online) (a) and (b) are simulation snapshots of the two surface phases. They are for the bulk field $h / k T=-0.075$, which is where these two phases coexist. (a) is the spin-down surface phase, and (b) is the spin-up surface phase. The phases are separated by what is called (in the language of fluids) a prewetting transition. For clarity only the first four layers $(z \leq 4)$ are shown. The surface is shown as a solid monolayer of dark red cubes while the lattice sites with up spins are shown as pale cubes. Down spins are not shown for clarity.

\section{EQUILIBRIUM BEHAVIOR}

The bulk behavior of the three-dimensional Ising model is very well understood. There is a bulk paramagnet-toferromagnet transition at $J / k T=0.222$ and $h / k T=0 .{ }^{16}$ This is illustrated in the schematic phase diagram of Fig. 3. The bulk transition is the vertical solid line at $h / k T=0$, ending at the bulk critical point. Below the critical point there is coexistence at $h / k T=0$ between spin-up and spin-down ferromagnetic phases. The surface phase behavior is much more complicated and is not fully known. ${ }^{4-6}$ It depends on four parameters: $J / k T, h / k T, J_{s} / k T$, and $h_{s} / k T$, while the bulk behavior just depends on the first two of these.

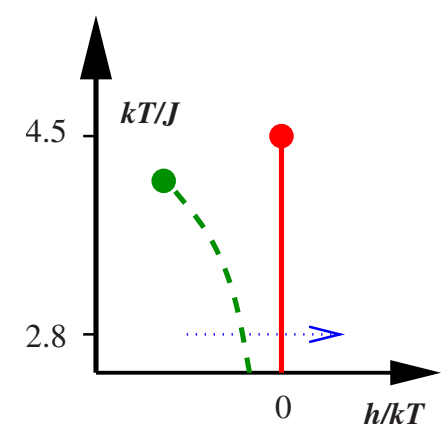

FIG. 3. (Color online) A schematic phase diagram for a three-dimensional Ising model. It shows both the bulk (solid vertical line) and surface (dashed curve) phase transitions. Both transitions end at critical points, indicated by circles. The pathway taken in our set of simulations is indicated by the dotted line and arrow.

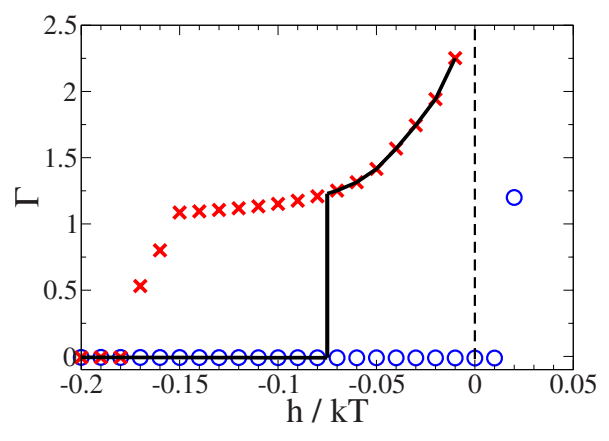

FIG. 4. (Color online) Isotherms of $\Gamma$ as a function of $h / k T$. The blue circles and red crosses are for increasing and for decreasing $h / k T$, respectively. The black curve is the equilibrium isotherm, with a first-order surface transition at $h / k T=-0.075$. On decreasing $h$, the surface spin-up phase becomes unstable at $h / k T=-0.16$, as can be seen by the drop in $\Gamma$. On increasing $h$, the surface spin-down phase becomes unstable at $h / k T=+0.02$, as can be seen by the jump in $\Gamma$.

This work was originally inspired by work on hard spheres, in which our best estimate ${ }^{9,14}$ suggests that the bulk crystal phase wets the wall/fluid interface and that there is hysteresis before wetting - suggesting a first-order surface transition. Thus we want an Ising model in which on increasing $h$, the sequence of behavior is surface phase transition, wetting, and then a bulk phase transition. Wetting is where at coexistence, one bulk phase is interposed between a surface and the other bulk phase. See the reviews ${ }^{20,21}$ for introductions to surface phase behavior. If there is wetting by the spin-up phase, as coexistence is approached on the spindown side of the phase transition, the thickness of the layer of the spin-up phase adsorbed at the surface diverges. This is measured by the excess number of up spins per surface lattice site, $\Gamma$. Here excess means the difference between the number of up spins with a surface, and the number in the same volume but without the surface.

We vary only $h$, keeping $J, J_{s}$, and $h_{s}$ constant. We work at a bulk coupling $J / k T=0.35$, a surface coupling $J_{s} / k T$ $=0.65$, and a surface field $h_{s} / k T=0.38$. All our results are for these values of $J, J_{s}$, and $h_{s}$. $\Gamma$ as a function of $h / k T$ is shown in Fig. 4. The jump in $\Gamma$ at the first-order surface transition is clear, as is the divergence in $\Gamma$ as $h \rightarrow 0$ that is characteristic of wetting. The surface phase transition is from a phase with $\Gamma$ close to 0 to one with $\Gamma$ close to 1 , i.e., to a surface phase in which approximately a monolayer of adsorbed spins is found at the surface. Snapshots of simulations of both phases are shown in Fig. 2.

Note that we generate a strongly first-order surface transition by using a large value of $J_{S}$ (almost twice $J$ ) and that the surface field strongly favors up spins in the surface layer of spins. To understand the surface behavior, consider the $J$ $=0$ limit. Then the surface monolayer of spins decouples from the bulk and undergoes a two-dimensional Ising paramagnet-to-ferromagnet transition at $J_{s} / k T=0.44$ and $h$ $+h_{s}=0 .{ }^{22}$ For temperatures below this the surface monolayer is in the spin-down phase for $h+h_{s}<0$ and in the spin-up phase for $h+h_{s}>0$.

When $J \neq 0$ the surface monolayer is now coupled to the bulk. This does not destroy the surface phase transition but it does shift it. At our values of $J, J_{s}$, and $h_{s}$, it occurs at 
$h / k T=h^{(s)} / k T=-0.075$. This value was obtained both by finding the point at which the forward and back nucleation rates in a relatively small system are equal, and by altering the boundary conditions to remove hysteresis and simulating the system. Finally, note that $J / k T=0.35$ is above the roughening temperature, ${ }^{6,23}$ which is estimated to be close to $J / k T=0.4$.

In the schematic phase diagram of Fig. 3, the line of prewetting transitions is shown by the dashed curve. Note that Fig. 3 is purely schematic; the line of prewetting transitions must end at a prewetting critical point ${ }^{20}$ but the temperature of this point may be above or below the bulk critical point. As the prewetting transition ends at a critical point, then formation of a wetting layer does not have to be via nucleation; if $T$ and $h$ are varied so as to take the system around the dashed curve, then the wetting layer will appear continuously.

\section{NUCLEATION}

Before we explicitly calculate nucleation rates, let us consider hysteresis. To study this we first started with a system at a field $h / k T=-0.2$. This was equilibrated in the spindown phase. The field $h / k T$ was then increased in a succession of steps, each with a size of 0.01. After each step we equilibrated at the new value of $h / k T$ for 10000 cycles and then averaged for another 10000 cycles to obtain a value for $\Gamma$. The values of $\Gamma$ are plotted as blue circles in Fig. 4. Note that the prewetting transition did not occur at all; the system remained with the surface in the spin-down surface phase until $h / k T=0.02$, at which point the bulk spin-up phase nucleated.

To observe hysteresis in the other direction, we started with a system with $h / k T$ slightly negative but with the surface spin-up phase present. We set $h / k T$ slightly negative because at $h=0$ the wetting layer has divergent thickness, and this would not fit into our simulation box. We then decreased $h / k T$ in steps of 0.01 , equilibrating and averaging as before, to obtain the red crosses in Fig. 4. Note that on the decreasing- $h$ isotherm when $h / k T$ reaches -0.16 , the surface adsorbed layer becomes unstable and evaporates. The spin-up surface phase becomes unstable here.

We used the FFS algorithm to calculate nucleation rates. We start with a configuration in which all spins are down, and then equilibrate in the metastable state at the required value of $h / k T$. Thus our starting states resemble the snapshot of Fig. 2(a). At each value of $h / k T$, we performed 5 runs and in each run there were 3000 configurations at each interface. A FFS run consists of first determining the flux leaving phase $A$ and storing 3000 configurations that have left $A$. Then the probability of systems starting from these configurations going forward toward phase $B$ is calculated in a stepwise manner. See Refs. 17 and 19 for details. We obtained the mean and standard deviations of the logs of the rates. The results are plotted in Fig. 5. We checked which phase the final configuration will grow into, by running on a simulation starting from this configuration. In all cases it grew into a surface wetting layer for $h<0$ and into a bulk spin-up phase for $h$ $>0$. We are not aware of any study of nucleation at a surface

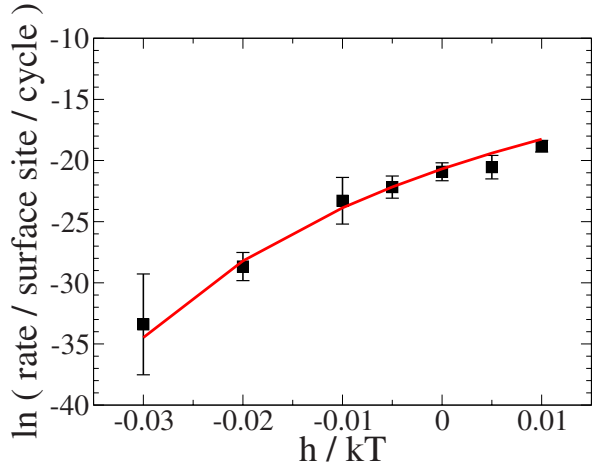

FIG. 5. (Color online) The natural logarithm of the nucleation rate per surface lattice site plotted as a function of $h / k T$. A cycle is one attempted spin flip per lattice site. Each point is the average of the logs of the rates from five runs. The error bars are the standard deviations of logs of the rates. The curve is a fit of a function of the form $-C /(h+0.075)$ to the logs of the rates; $C=1.55$.

in this model, although Pan and Chandler ${ }^{24}$ studied nucleation in the bulk of the three-dimensional Ising model.

No discontinuity in either the rate or its derivative is visible at $h=0$. Indeed the nucleation rate is well fit by a continuous function (red curve) of the form of classical nucleation theory for two-dimensional nucleation. Snapshots of nuclei near the top on the barrier on either side of $h=0$ are shown in Fig. 1: there is no obvious significant difference between the two. From runs at $h / k T=-0.005$ and 0.005 , we obtained 142 and 124 critical nuclei, respectively. Here a critical nucleus is defined as being one that has a value of the committor between 0.495 and 0.505 . The committor for a spin configuration is the probability that in an unconstrained simulation started from this configuration, the simulation reaches phase $B$ (the nucleating phase) before phase $A$. To determine the committor we run 200 simulations from each potential critical nucleus configuration.

At $h / k T=-0.005$ the number of up spins in the critical nuclei configurations was $490 \pm 20$ (mean \pm standard) deviation. Here $\lambda_{A}$, the maximum number of up spins in the metastable phase, was set at 370 . Thus, the mean increase in the number of up spins required to reach the critical configuration is 101 . At $h / k T=0.005$ the number of up spins in the critical nuclei configurations was $472 \pm 17$. Here $\lambda_{A}$, the maximum number of up spins in the metastable phase, was set at 380. Thus, the average increase in the number of up spins in the critical configuration is 92 . The numbers of up spins in the surface layer were $116 \pm 7$ and $103 \pm 8$ at $h / k T=-0.005$ and 0.005 , respectively, again very similar numbers. Thus we conclude that although as $h$ increases beyond zero, the phase that is nucleating changes, the nucleus near the top of the barrier does not "know" this, and so is unaffected.

It is interesting to note that the nucleation rate precisely at coexistence is a finite, nonzero number. A naive application of classical nucleation theory would suggest that here the nucleation barrier for the new bulk phase would be zero if there is wetting, or infinite if the contact angle is nonzero. Liquid mixtures, such as methanol/cyclohexane, ${ }^{20,25}$ have been studied, which have phase diagram analogous to that of our model. Experiments have varied the temperature while 
remaining at coexistence. They observed hysteresis: the product of finite nucleation rates. Thus our finding of a nonzero, finite nucleation rate at coexistence is not novel. However, our results may be useful in understanding the microscopic physics that underlies these experimental observations. As our study is the first simulation study, it provides the first exact results for the nucleus of a new wetting layer. Earlier theoretical work is reviewed by Bonn and Ross. $^{20}$

Finally, we note that if we start with a system with $h$ large and negative and increase $h / k T$ at some fixed rate, then depending on this rate the surface may or may not have time to nucleate before $h$ crosses 0 . If $h / k T$ is increased at a rate slower than $\sim \exp (-20) L^{2}$, then the surface spin-up phase will have time to nucleate. As wetting then occurs, as $h$ crosses 0 there is then no further nucleation barrier to the formation of the bulk spin-up phase. In contrast, if $h / k T$ is increased at a rate faster than $\sim \exp (-20) L^{2}$, then the surface spin-up phase does not have time to nucleate before $h$ crosses 0 . Then the surface spin-up phase will not have time to form, and there will be a barrier to nucleation of the bulk spin-up phase.

In this section we have considered nucleation of the spin-up surface and bulk phases. Of course, there is the reverse problem of the nucleation of the spin-down phase at a surface with a pre-existing wetting layer of up spins. If there is a thick wetting layer, the barrier for this may be very large; see the review of Bonn and Ross ${ }^{20}$ for a discussion of this problem. Thus the two nucleation problems, nucleation of the spin-up and of the spin-down phases, may, at least under some circumstances, be very different. We leave nucleation of the spin-down phase to future work.

\section{A. Comparison with classical nucleation theory}

The classical nucleation theory prediction for the nucleation rate of a surface transition can easily be obtained. Classical nucleation theory assumes that the rate can be expressed as ${ }^{1,26}$

$$
\text { rate }=\nu \exp \left(-\Delta F^{*} / k T\right)
$$

where $\nu$ is a kinetic prefactor that is assumed to vary only weakly with $h$, and $\Delta F^{*}$ is the free-energy barrier to nucleation.

In two dimensions the free energy of a nucleating domain of a new phase looks like

$$
\Delta F(R)=-\pi R^{2} t\left(h-h^{(s)}\right)+2 \pi R \tau .
$$

Here, the nucleus is assumed to be circular with radius $R$, and $t$ layers thick on average. Then the first term in Eq. (3) is simply the volume of the nucleus times the free energy per site, assumed to be linear in $h$ (and 0 at $h=h^{(s)}$ - which it must be). The second term comes from the free-energy cost of the interface at the edge of the circular nucleus, which is equal to the length of this interface times the line tension $\tau$. The barrier $\Delta F^{*}$ is simply the maximum of $\Delta F$ as a function of $R$, which is

$$
\Delta F^{*}=\frac{\pi \tau^{2}}{t\left(h-h^{(s)}\right)} .
$$

Thus we see that the functional form of log of the rate is $-C /\left(h-h^{(s)}\right)$, where $C$ is a constant. We have fitted a function of this form to our simulation data (see Fig. 5). The quality of the fit is excellent. Note that for three-dimensional nucleation, the functional form is $-C /\left(h-h^{(s)}\right)^{2}$ and that this provides only a very poor fit to the data (fit not shown). Thus we conclude that from the perspective of classical nucleation theory, nucleation of both phases looks like nucleation of a new surface phase.

\section{B. Hard spheres at a hard wall}

This work was inspired by work on fluids of hard spheres near their fluid-to-solid bulk transition, and in contact with a smooth hard wall. Hard spheres freeze via a strongly first-order phase transition from a fluid with a reduced density $\rho_{f}=0.943$ (Ref. 27) to a crystal with a density about $10 \%$ higher. This bulk behavior is well understood. However, the behavior of hard spheres near a hard wall has been harder to determine. In 1992 Courtemanche and co-workers ${ }^{7,8}$ concluded that the crystal phase of hard spheres wets a hard wall, i.e., as a fluid of hard spheres is compressed up to a density $\rho_{f}$, a layer of the crystalline phase forms at the hard wall whose thickness diverges at $\rho_{f}$. Dijkstra $^{9}$ also found this. See also Dijkstra and van Roij. ${ }^{10}$ Moreover, she found hysteresis suggesting that the first crystalline layer formed via a first-order prefreezing, surface transition. These simulations were performed at and below $\rho_{f}$, where there is no bulk crystal phase.

Heni and Löwen, ${ }^{28}$ then Laird and co-workers ${ }^{12-14}$ took an alternative route to determining whether or not the crystal phase wets the fluid/wall interface. They determined all three relevant interfacial tensions, here $\gamma_{w 111}, \gamma_{111 f}$, and $\gamma_{w f}$. These are the interfacial tensions of the wall/(111)-crystal-plane, (111)-crystal-plane/fluid, and wall/fluid interfaces, respectively. Then there is wetting if $\gamma_{w 111}+\gamma_{111 f}<\gamma_{w f}$. The early calculations of these tensions ${ }^{12,13,28}$ found that the left- and right-hand sides of this inequality were so close to each other that the results were not sufficiently accurate to answer the question one way or the other. However, the most recent calculations by Laird and co-workers, ${ }^{13,14}$ and by Fortini and Dijkstra, ${ }^{11}$ have more-or-less settled the issue: the crystal wets the wall-fluid interface. The surface tension of interfaces involving the crystal varies depending on the plane of the crystal lattice that lies in the plane of the interface. Wetting occurs with (111) lattice planes in contact with both the wall and the fluid.

Thus, we conclude that the evidence is that there is both wetting by the crystalline phase of the wall/fluid interface, and that there is a first-order surface transition at which a layer of spheres at the wall freezes. This transition is called either prefreezing or precrystallization. However, Auer and Frenkel $^{15}$ studied nucleation of the crystal phase at a hard wall and found a nucleation barrier at a pressure beyond the coexistence pressure. A naive application of classical nucleation theory would suggest that as the crystal phase wets the fluid/wall interface, there should be no nucleation barrier. ${ }^{1,26}$ 
However, we also have wetting in our Ising model and we too have a nucleation barrier for the bulk phase. This suggests that hard spheres near a hard wall and our Ising model may have qualitatively the same nucleation behavior. This would imply that the nucleation barrier to crystallization may be nonzero and finite actually at fluid-crystal coexistence. This would be in the absence of prenucleated surface crystal layer. It also implies that the nucleation barrier will be a smooth function of density as $\rho_{f}$ is crossed.

\section{CONCLUSION}

Within the precision with which can determine it numerically, the nucleation rate of our Ising model varies smoothly across the bulk phase transition. This is despite the fact that at the transition the phase that is nucleating changes. Our assumption is that this due to the fact that the critical nucleus is small, the nuclei in Fig. 1 are on average less than two layers thick. Therefore the nucleus has no means of "knowing" whether it will grow into a wetting layer that is many times its own thickness but finite, or into an infinite bulk phase. If this understanding is correct, our finding will be generic and apply generally to systems where nucleation of the bulk phase occurs at a surface that undergoes a firstorder surface transition.

In the previous section we have discussed one system to which our findings may apply, namely, hard spheres at a hard wall. Another set of systems with analogous phase diagrams are liquids and liquid mixtures at walls. Experiments on liquid mixtures have found prewetting transitions; they found hysteresis that is similar to that seen in our Fig. 4. See, for example, the study of Kellay et al. ${ }^{29}$ of methanol/ cyclohexane mixtures. Work on these systems is covered in the review of Bonn and Ross. ${ }^{20}$ Liquid mixtures at surfaces may be the best system to observe the effects seen here in experiment. For example, the waiting time before a liquid layer or bulk liquid appears could be determined across the bulk condensation transition.

\section{ACKNOWLEDGMENTS}

It is a pleasure to thank A. Page for useful discussions.

${ }^{1}$ R. P. Sear, J. Phys.: Condens. Matter 19, 033101 (2007).

${ }^{2}$ D. Chandler, Introduction to Modern Statistical Mechanics (Oxford University Press, New York, 1987).

${ }^{3}$ M. Plischke and B. Bergersen, Equilibrium Statistical Physics (World Scientific, Singapore, 1994).

${ }^{4}$ K. Binder, D. P. Landau, and S. Wansleben, Phys. Rev. B 40, 6971 (1989).

${ }^{5}$ D. P. Landau and K. Binder, Phys. Rev. B 41, 4633 (1990).

${ }^{6}$ K. Binder and D. P. Landau, Phys. Rev. B 46, 4844 (1992).

${ }^{7}$ D. J. Courtemanche and F. van Swol, Phys. Rev. Lett. 69, 2078 (1992).

${ }^{8}$ D. J. Courtemanche, T. A. Pasmore, and F. van Swol, Mol. Phys. 80, 861 (1993).

${ }^{9}$ M. Dijkstra, Phys. Rev. Lett. 93, 108303 (2004).

${ }^{10}$ M. Dijkstra and R. van Roij, J. Phys.: Condens. Matter 17, S3507 (2005).

${ }^{11}$ A. Fortini and M. Dijkstra, J. Phys.: Condens. Matter 18, L371 (2006).

${ }^{12}$ R. L. Davidchack and B. B. Laird, Phys. Rev. Lett. 85, 4751 (2000).

${ }^{13}$ R. L. Davidchack, J. R. Morris, and B. B. Laird, J. Chem. Phys. 125, 094710 (2006).

${ }^{14}$ B. B. Laird and R. L. Davidchack, J. Phys. Chem. C 111, 15952 (2007).

${ }^{15}$ S. Auer and D. Frenkel, Phys. Rev. Lett. 91, 015703 (2003).

${ }^{16}$ A. L. Talapov and H. W. J. Blöte, J. Phys. A 29, 5727 (1996).

${ }^{17}$ R. J. Allen, P. B. Warren, and P.-R. ten Wolde, Phys. Rev. Lett. 94, 018104 (2005).

${ }^{18}$ R. J. Allen, D. Frenkel, and P. R. ten Wolde, J. Chem. Phys. 124, 024102 (2006).

${ }^{19}$ R. P. Sear, J. Phys. Chem. B 110, 4985 (2006).

${ }^{20}$ D. Bonn and D. Ross, Rep. Prog. Phys. 64, 1085 (2001).

${ }^{21}$ M. Schick, in Liquids at Interfaces, Les Houches XLVIII, edited by J. Charvolin, J. F. Joanny, and J. Zinn-Justin (Elsevier, Amsterdam, 1990).

${ }^{22}$ L. Onsager, Phys. Rev. 65, 117 (1944).

${ }^{23}$ M. Hasenbusch, S. Meyer, and M. Pütz, J. Stat. Phys. 85, 383 (1996).

${ }^{24}$ A. C. Pan and D. Chandler, J. Phys. Chem. 108, 19681 (200 4).

${ }^{25}$ D. Bonn, H. Kellay, and J. Meunier, Phys. Rev. Lett. 73, 3560 (1994).

${ }^{26}$ P. G. Debenedetti, Metastable Liquids (Princeton University Press, Princeton, 1996).

${ }^{27}$ W. W. Wood and J. D. Jacobson, J. Chem. Phys. 27, 1207 (1957); B. J. Alder and T. E. Wainwright, ibid. 27, 1208 (1957); W. G. Hoover and F. H. Ree, ibid. 49, 3609 (1968).

${ }^{28}$ M. Heni and H. Löwen, Phys. Rev. E 60, 7057 (1999).

${ }^{29}$ H. Kellay, D. Bonn, and J. Meunier, Phys. Rev. Lett. 71, 2607 (1993). 